https://doi.org/10.22319/rmcp.v11i4.5192

Nota de investigación

\title{
Efecto de la adición de clorhidrato de zilpaterol genérico en el perfil bioquímico y hematológico de ovinos de pelo engordados en corral
}

Arnulfo Vicente Pérez ${ }^{\text {a† }}$

Leonel Avendaño Reyes ${ }^{b}$

Ulises Macías Cruz ${ }^{\mathrm{b}}$

Antonio Aguilar Quiñones ${ }^{a}$

Ricardo Vicente Pérez ${ }^{c}$

Miguel Mellado Bosque ${ }^{d}$

Miguel Ángel Gastélum Delgado ${ }^{\mathrm{e}}$

Abelardo Correa Calderón ${ }^{\mathrm{b}}$

G. López-Rincón ${ }^{f}$

Rubén Barajas Cruz ${ }^{\text {a }}$

Juan Eulogio Guerra Liera ${ }^{e^{*}}$

${ }^{a}$ Universidad Autónoma de Sinaloa. Facultad de Medicina Veterinaria y Zootecnia, Culiacán, Sinaloa, México.

${ }^{\mathrm{b}}$ Universidad Autónoma de Baja California. Instituto de Ciencias Agrícolas. Ejido Nuevo León, Valle de Mexicali, Baja California, México.

${ }^{c}$ Universidad de Guadalajara. Centro Universitario de la Costa Sur. Autlán de Navarro, Jalisco, México.

${ }^{d}$ Universidad Autónoma Agraria Antonio Narro. Departamento de Nutrición Animal. Saltillo, Coahuila, México.

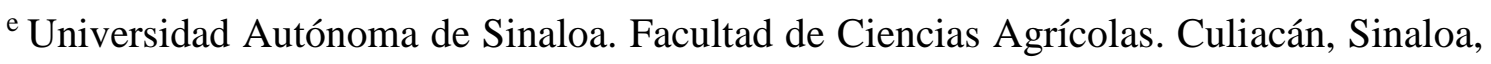
México.

${ }^{\mathrm{f}}$ Laboratorios Virbac México, S.A. de C.V., Zapopan, Jalisco, México. 
*Autor de correspondencia: dr.juanguerral@ gmail.com

${ }^{\dagger}$ Alumno del Doctorado en Ciencias Agropecuarias, FMVZ-UAS.

\section{Resumen:}

El objetivo fue evaluar la adición de clorhidrato de zilpaterol (CZ, genérico) en la dieta de ovinos de pelo de engorda, sobre variables hematológicas y bioquímicas como indicadores del estado de salud. Treinta y dos (32) corderos de pelo cruzados (Dorper $\mathrm{x}$ Pelibuey) se bloquearon por peso inicial y cuatro tratamientos y se asignaron aleatoriamente dentro de cada bloque: $\mathrm{T} 1=$ dieta basal (grupo testigo), $\mathrm{T} 2=$ dieta basal más $0.10 \mathrm{mg} \cdot \mathrm{kg}^{-1}$ de PV d${ }^{-1}$ de CZ (Grofactor ${ }^{\circledR}$, Virbac México, Guadalajara, México), $\mathrm{T} 3=$ dieta basal más $0.20 \mathrm{mg} \cdot \mathrm{kg}^{-1}$ de $\mathrm{PV} \mathrm{d}{ }^{-1}$ de CZ y T4= dieta basal más $0.30 \mathrm{mg} \cdot \mathrm{kg}^{-1} \mathrm{de}$ $\mathrm{PV} \mathrm{d}^{-1}$ de CZ. Se tomaron muestras de sangre los días 1, 15 y 30 del estudio. El perfil hematológico se estimó en muestras de sangre fresca, mientras que metabolitos, electrolitos y hormonas en muestras de suero. El diseño experimental fue bloques completos al azar y se efectuó análisis de polinomios ortogonales para determinar la tendencia de las respuestas a los niveles de CZ. Los niveles de colesterol y urea fueron mayores $(P<0.05)$ en T3 que en T2; asimismo, el nivel de concentración corpuscular medio de hemoglobina fue mayor $(P<0.05)$ en $\mathrm{T} 1$ que en $\mathrm{T} 3$, mientras que el ancho de distribución de eritrocitos fue mayor $(P<0.05)$ en T2 y T3 que en T4. Los niveles de Na y el número de plaquetas mostraron tendencia lineal $(P<0.05)$ a disminuir y aumentar, respectivamente, conforme los niveles de CZ aumentaron. Se observó tendencia cuadrática $(P<0.05)$ en la concentración corpuscular media de hemoglobina y ancho de distribución de eritrocitos al aumentar la dosis de CZ (genérico). Las variables restantes no mostraron tendencias significativas a los niveles de CZ (genérico). Los valores de los perfiles bioquímico y hematológico se encontraron dentro del rango de referencia, lo que sugiere que la adición de CZ no alteró el estado de salud de corderos en engorda.

Palabras clave: Hemoglobina, Corderos, Metabolitos, Electrolitos, Clorhidrato de zilpaterol.

Recibido: 23/12/2018

Aceptado: 25/09/2019

La ganadería ovina destinada a producir carne en México crece en forma considerable, pero la demanda de animales para abasto es mayor a lo que se produce, lo que se traduce en importaciones de ganado en pie y en canal de Estados Unidos, Nueva Zelanda y Australia, principalmente ${ }^{(1)}$. Los ovinos de pelo han sido una alternativa para cubrir estas demandas de carne porque requieren menos cuidados que las razas de lana. Además, tienen la peculiaridad de adaptarse, reproducirse y producir bajo cualquier sistema de producción. Por mostrar baja estacionalidad reproductiva, esta especie mantiene la producción de cordero durante todo el año; sin embargo, tiene la desventaja de su limitada 
ganancia de peso $^{(2)}$. Por esto, los engordadores complementan usando promotores del crecimiento, que permiten obtener animales más eficientes para producir carne ${ }^{(3)}$. Los agonistas adrenérgicos-beta (AA- $\beta$ ) son una atractiva estrategia que ha impactado positivamente la producción de ovinos en engorda ${ }^{(4)}$.

Comparando resultados de distintos AA- $\beta$ (clorhidrato de zilpaterol, clorhidrato de ractopamina, terbutalina, isoprotenerol, etc.), se concluyó que el clorhidrato de zilpaterol (CZ) es buena opción en la engorda de ovinos ${ }^{(5)}$. No obstante, los resultados observados son contradictorios en términos de rendimiento ${ }^{(4,6)}$ y características de la canal ${ }^{(6,7)}$. Dado que la adición de $\mathrm{CZ}$ en la dieta promueve cambios fisiológicos, metabólicos, hormonales y hematológicos, se puede comprometer la salud del animal ${ }^{(8)}$. Además, el uso de AA- $\beta$ en rumiantes y sus efectos negativos en el bienestar animal han sido de las preocupaciones más importantes en el sector agropecuario ${ }^{(9)}$. Los efectos de la adición del CZ en la dieta se han centrado en el rendimiento animal y características de la canal, mientras que los efectos de este compuesto en la salud y bienestar se atienden de forma trivial ${ }^{(10)}$. A pesar de que se ha encontrado mayor morbilidad en bovinos suplementados con AA- $\beta$ en la finalización de la engorda ${ }^{(11)}$, existen pocos estudios que evalúen su efecto en la salud animal y que incluyan el análisis de componentes bioquímicos y hematológicos. El objetivo de este estudio fue evaluar el efecto de adicionar distintas dosis del AA- $\beta$ genérico $\mathrm{CZ}$ sobre variables hematológicas y bioquímicas en ovinos de pelo finalizados en corral.

El estudio se realizó en la época otoño-invierno del 2015 en la Unidad Experimental Ovina del Instituto de Ciencias Agrícolas de la Universidad Autónoma de Baja California, en el Valle de Mexicali, Baja California, México $\left(32.8^{\circ} \mathrm{N}, 114.6^{\circ} \mathrm{O}\right)$. Las condiciones climáticas de esta región son tipo Desierto de Sonora, definidas por un clima extremadamente seco y cálido, con temperaturas máximas en verano $\geq 42{ }^{\circ} \mathrm{C}$ y mínimas en invierno $\leq 0{ }^{\circ} \mathrm{C}$; la precipitación pluvial anual promedia $85 \mathrm{~mm}^{(12)}$.

Se utilizaron 32 corderos de pelo F1 cruza de Dorper $x$ Pelibuey con peso promedio de $29.3 \pm 0.22 \mathrm{~kg}$ y edad entre 5 y 6 meses. Se formaron grupos de cuatro corderos por peso inicial y se asignaron aleatoriamente a uno de cuatro tratamientos: $\mathrm{T} 1=$ dieta basal (grupo testigo); T2= dieta basal más $0.10 \mathrm{mg} \cdot \mathrm{kg}^{-1}$ de PV d ${ }^{-1}$ de CZ (Grofactor ${ }^{\circledR}$, Virbac México, Guadalajara, México); T3= dieta basal más $0.20 \mathrm{mg} \cdot \mathrm{kg}^{-1}$ de $\mathrm{PV} \mathrm{d} \mathrm{d}^{-1}$ de $\mathrm{CZ}$ y T4= dieta basal más $0.30 \mathrm{mg} \cdot \mathrm{kg}^{-1}$ de $\mathrm{PV} \mathrm{d}^{-1}$ de $\mathrm{CZ}$. Los corderos se alojaron en corraletas individuales $(1.0 \times 1.5 \mathrm{~m})$ provistas de comederos, bebedero y sombra. El alimento se ofreció dos veces por día (0700 y 1500 h) en proporción 40:60. La ración consistió en grano de trigo molido (60\%), heno de alfalfa (17.5\%), paja de trigo (11\%), harina de soya $(7 \%)$, aceite de soya ( $2 \%)$, piedra caliza (1\%), fosfato dicálcico $(1 \%)$ y $0.5 \%$ de sal común, la cual se formuló para aportar $15 \%$ de PC y $2.9 \mathrm{Mcal}$ de $\mathrm{EM} \mathrm{kg}^{-1} \mathrm{de}_{\mathrm{MS}}{ }^{(13)}$. Para garantizar el consumo del CZ, la dosis diaria del producto se mezcló en $30 \mathrm{~g}$ de grano de trigo molido y se ofreció por la mañana antes de servir la dieta basal. En el día 30 de la prueba, el CZ se retiró siguiendo con las indicaciones del fabricante del producto. El experimento duró 47 días (15 de adaptación, 30 de engorda y 2 de retiro). 
Para analizar metabolitos, electrolitos y componentes hematológicos se colectaron muestras de sangre en tubos vacutainer de 10 y $4 \mathrm{ml}$ mediante punción en la vena yugular. La sangre se colectó antes de ofrecer la dieta por la mañana $(0600$ h) tres veces: fase inicial, intermedia y final de la engorda (días 1, 15 y 30). Se utilizaron muestras de sangre fresca para el análisis hematológico usando un equipo automatizado (Auto Hematology Analyzer, MINDRAY, BC-2800 Vet; Shenzhen, China). La sangre colectada en tubos de $10 \mathrm{ml}$ se centrifugó a $3,500 \mathrm{rpm}$ a $10^{\circ} \mathrm{C}$ por $15 \mathrm{~min}$; después se separó el suero por duplicado en viales de $2 \mathrm{ml}$ y se almacenaron a $-20{ }^{\circ} \mathrm{C}$ para el posterior análisis de los metabolitos glucosa (Glu), colesterol (Col), urea (Ur), triglicéridos (Trig) y proteína total $(\mathrm{PT})$, electrolitos $(\mathrm{Na}, \mathrm{K}$ y Cl$)$ y hormonas tiroxina $\left(\mathrm{T}_{4}\right)$ y triiodotironina $\left(\mathrm{T}_{3}\right)$. Los metabolitos se determinaron con un equipo de química sanguínea (Model DT-60, Johnson Co.; High Wycombe, UK), mientras que los electrolitos con un equipo automatizado (Electrolyte Analyzer LW E60A; Landwind Medical; Shenzhen, China). La determinación de hormonas se realizó con un equipo automatizado para pruebas de Elisa y de Quimioluminiscencia (CLIA) marca Thunderbolt@ Analyzer (Davis, CA, USA).

Las respuestas se analizaron bajo un diseño de bloques completamente al azar. Se realizó un análisis de polinomios ortogonales para determinar la tendencia en las respuestas a través de los niveles del AA- $\beta$. Se declararon significancias a probabilidad de $P \leq 0.05$ mediante el uso del PROC MIXED de SAS. Dado que la tendencia cúbica no resultó significativa para ninguna variable analizada, se omitió de los cuadros de resultados. Todos los datos se procesaron en el paquete estadístico Statistical Analysis System ${ }^{(14)}$.

El Cuadro 1 muestra los resultados de la adición de dosis de $\mathrm{CZ}$ sobre metabolitos sanguíneos en corderos de engorda. Los niveles de Col y Ur fueron mayores $(P<0.05)$ en T3 que en T2. Los niveles de Trig, Glu y PT no fueron afectados $(P>0.05)$ por la adición de CZ. Estos valores obtenidos se encuentran dentro del rango de referencia para ovinos $^{(15,16)}$. Resultados similares reportaron López-Carlos et al ${ }^{(6)}$ al no encontrar diferencias en PT, Glu ni Trig al suplementar dosis similares de CZ a las del presente estudio usando ovinos cruzados Dorper x Katahdin. Otros autores ${ }^{(17)}$ reportaron que la adición de $0.20 \mathrm{mg} \cdot \mathrm{kg}^{-1}$ de PV día ${ }^{-1}$ de $\mathrm{CZ}$ a ovinos de lana no modificó los niveles sanguíneos de Glu, Trig, PT ni Col, encontrando sólo una disminución en los niveles de Ur. También se ha mencionado que la administración de $\mathrm{CZ}$ en bovinos no modificó los metabolitos sanguíneos ${ }^{(8,18)}$. Hatefi et $a l^{(19)}$, en machos caprinos encontraron que la adición de CZ disminuyó los niveles plasmáticos de Glu y Col, sin afectar los niveles de Trig.

La administración de AA- $\beta$ tiene como consecuencia inmediata un incremento en la gluconeogénesis, lo que a su vez produce un aumento inminente de los niveles séricos de $\mathrm{Glu}^{(8)}$; sin embargo, se estima que el aumento inicial en las concentraciones de Glu desaparece conforme se incrementa el tiempo de administración, hasta retornar a concentraciones normales por una disminución de sensibilidad tisular ${ }^{(20)}$, efecto que pudo haberse presentado en el presente estudio, donde los niveles de metabolitos retornaron a sus concentraciones normales. Esto se afirma por el hecho de que los metabolitos 
asociados al estado energético y proteico no fueron afectados por la adición de diferentes dosis de CZ en la dieta, indicando que los corderos no modificaron ni comprometieron su homeostasis ni metabolismo. Por tanto, es posible considerar, de forma inicial, que el uso del AA- $\beta$ Grofactor ${ }^{\circledR}$ no tuvo efectos perjudiciales para la salud de los ovinos. Sin embargo, a la fecha son pocos los estudios realizados sobre el efecto de AA- $\beta$ en los metabolitos sanguíneos, y más aún, los resultados obtenidos aún son inconsistentes ${ }^{(19)}$.

Cuadro 1: Concentraciones séricas de metabolitos en ovinos de pelo en respuesta a distintas dosis de clorhidrato de zilpaterol

\begin{tabular}{llllllll}
\hline \multicolumn{4}{c}{ Dosis de CZ $\left(\mathrm{mg} \cdot \mathrm{kg}^{-1} \mathrm{de} \mathrm{PV} \mathrm{día}^{-1}\right)$} & EEM & \multicolumn{2}{c}{ Efectos } \\
\hline & 0 & 0.10 & 0.20 & 0.30 & & $\mathrm{~L}$ & $\mathrm{C}$ \\
\hline Col, mg/dl & $49.4^{\mathrm{ab}}$ & $46.7^{\mathrm{a}}$ & $55.4^{\mathrm{b}}$ & $50.5^{\mathrm{ab}}$ & 3.02 & 0.36 & 0.71 \\
Trig, mg/dl & 26.0 & 26.7 & 25.8 & 26.4 & 1.40 & 0.95 & 0.98 \\
Glu, mg/dl & 64.3 & 62.6 & 65.7 & 61.8 & 4.12 & 0.83 & 0.82 \\
PT, mg/dl & 6.80 & 6.85 & 6.82 & 7.03 & 0.108 & 0.17 & 0.47 \\
Urea, mg/dl & $38.3^{\text {ab }}$ & $35.4^{\mathrm{a}}$ & $40.9^{\mathrm{b}}$ & $38.5^{\mathrm{ab}}$ & 1.69 & 0.40 & 0.91 \\
\hline
\end{tabular}

${ }^{\mathrm{ab}}$ Promedios con distinta literal en la misma hilera difieren $(P<0.05)$.

$\mathrm{EEM}=$ error estándar de la media; $\mathrm{L}=$ lineal; $\mathrm{C}=$ cuadrática

Col $=$ colesterol $;$ Trig $=$ Triglicéridos $;$ Glu= Glucosa $; \mathrm{PT}=$ Proteína total .

Los resultados de suplementar distintas dosis de CZ en electrolitos sanguíneos y hormonas tiroideas se muestran en el Cuadro 2. La administración de CZ mostró reducción significativa $(P<0.01)$ en los niveles séricos de Na, mostrando además una tendencia lineal con el incremento de los niveles de $\mathrm{CZ}$ en la dieta. Los niveles séricos de $\mathrm{Cl}, \mathrm{K}, \mathrm{T}_{3}$ y $\mathrm{T}_{4}$ no fueron afectados $(P>0.05)$ por los distintos tratamientos. Los AA- $\beta$ se consideran excelentes redistribuidores de nutrientes a favor de la formación de músculo esquelético y reducción del depósito de grasa en la canal ${ }^{(21)}$, no obstante, es posible que afecten la concentración de diversos componentes bioquímicos involucrados en el desarrollo del tejido muscular y adiposo ${ }^{(22)}$. El efecto cuadrático observado en las concentraciones de $\mathrm{Na}$ se dio en función de los niveles crecientes de $\mathrm{CZ}$ en la dieta. La adición de $0.10 \mathrm{mg}$ de $\mathrm{CZ}$ causó un decremento de $\mathrm{Na}$ en el suero, mientras que la dosis de $0.20 \mathrm{mg}$ de $\mathrm{CZ}$ mantuvo los niveles séricos de Na. Por otro lado, la adición de $0.30 \mathrm{mg}$ de $\mathrm{CZ}$ disminuyó de forma considerable los niveles de este electrolito. A pesar de que se encontró diferencia significativa en los niveles de Na sérico entre dosis, todos los valores se encuentran dentro del rango de referencia normal para ovinos ${ }^{(15,16)}$, lo cual sugiere que los animales tratados mantuvieron su presión osmótica y equilibrio ácido-base en el organismo, sin síntoma importante de estrés alguno.

El K juega un papel importante en la regulación del agua dentro y fuera de la célula. Así, mantener niveles óptimos de $\mathrm{K}$ sérico es importante para el correcto funcionamiento del organismo. No obstante, Buntyn et al ${ }^{(23)}$ encontraron que la adición de CZ promovió la disminución de niveles sanguíneos de $\mathrm{K}$, resultados que pueden deberse a un aumento en el depósito de músculo magro durante la adición con $\mathrm{CZ}$. El presente estudio tuvo 
resultados similares, ya que mostraron que el nivel más bajo de K se presentó en el nivel más alto de CZ. También se observó que los ovinos no tratados tuvieron mayor concentración de $\mathrm{K}$ con respecto a los tratados con $0.30 \mathrm{mg} \cdot \mathrm{kg}^{-1}$. Frese et al ${ }^{(8)}$ reportaron que la adición de $\mathrm{CZ}$ y clorhidrato de ractopamina no modificaron los niveles de $\mathrm{K}$ con respecto al testigo en novillos en finalización. Aunque no se ha reportado ningún estudio donde la ingestión de AA- $\beta$ cause disminución de $\mathrm{Na}$ plasmático en ovinos, en ganado equino se reportó que la disminución de Na sanguíneo por efecto de AA- $\beta$ puede darse en casos muy extremos, ya sea por la pérdida durante la sudoración profusa o por deterioro del transporte renal de Na; incluso, la hipocloremia puede estar asociada con la liberación endógena de glucocorticoides o insuficiencia renal ${ }^{(24)}$, efecto que posiblemente no ocurrió en el presente estudio. Por su parte, otros trabajos ${ }^{(8,23)}$ reportan que novillos y vaquillas en finalización de la engorda tuvieron similares concentraciones de $\mathrm{Na}$ al adicionar los AA- $\beta$ CZ y clorhidrato de ractopamina en la dieta, resultados que fueron atribuidos a la disponibilidad y consumo a libre acceso de agua de los animales.

Cuadro 2: Concentraciones séricas de electrolitos y hormonas tiroideas en ovinos de pelo en respuesta a distintas dosis de clorhidrato de zilpaterol

\begin{tabular}{llllllll}
\hline & \multicolumn{3}{c}{ Dosis de CZ $\left(\mathrm{mg} \cdot \mathrm{kg}^{-1} \mathrm{de} \mathrm{PV} \mathrm{día}^{-1}\right)$} & \multicolumn{2}{c}{ EEM } & \multicolumn{2}{c}{ Tendencia } \\
\hline & 0 & 0.10 & 0.20 & 0.30 & & $\mathrm{~L}$ & $\mathrm{C}$ \\
\hline $\mathrm{Na}, \mathrm{mmol}$ & $139.7^{\mathrm{a}}$ & $138.2^{\mathrm{ab}}$ & $138.5^{\mathrm{ab}}$ & $137.1^{\mathrm{b}}$ & 0.584 & $<0.01$ & 0.94 \\
$\mathrm{Cl}, \mathrm{mmol}$ & $113.3^{\mathrm{a}}$ & 114.3 & $113.3^{\mathrm{a}}$ & 113.9 & 0.489 & 0.95 & 0.84 \\
$\mathrm{~K}, \mathrm{mmol}$ & $6.29^{\mathrm{a}}$ & $5.85^{\mathrm{ab}}$ & $6.31^{\mathrm{a}}$ & $5.57^{\mathrm{b}}$ & 0.181 & 0.10 & 0.51 \\
$\mathrm{~T}_{3}, \mathrm{ng} / \mathrm{ml}$ & 1.40 & 1.19 & $1.32^{2}$ & 1.25 & 0.728 & 0.33 & 0.38 \\
$\mathrm{~T}_{4} \mathrm{ng} / \mathrm{ml}$ & 2.92 & 3.06 & 2.40 & 2.29 & 0.343 & 0.10 & 0.74 \\
\hline
\end{tabular}

$\mathrm{EEM}=$ error estándar de la media; $\mathrm{L}=$ lineal; $\mathrm{C}=$ cuadrática.

$\mathrm{K}=$ potasio; $\mathrm{Na}=$ sodio $; \mathrm{Cl}=$ cloro; $\mathrm{T}_{3}=$ triiodotironina; $\mathrm{T}_{4}=$ tiroxina.

ab Promedios con distinta literal en la misma hilera difieren $(P<0.05)$.

Se ha encontrado que las hormonas $\mathrm{T}_{4} \mathrm{y}_{3} \mathrm{~T}_{3}$ no muestran variación durante tratamientos agudos o crónicos con AA- $\beta^{(25)}$, resultados que coinciden con los del presente estudio al no encontrar efectos por la adición de CZ durante 30 días. No obstante, Hatefi et al ${ }^{(19)}$ reportaron que los niveles de $\mathrm{T}_{3}$ y $\mathrm{T}_{4}$ aumentaron después de la administración crónica de distintos AA- $\beta$ en caprinos, resultados que pudieron haberse dado por el aumento en la lipólisis. Las hormonas tiroideas se relacionan ampliamente con aspectos del metabolismo de hidratos de carbono, como la absorción y movilización de glucosa hacia el tejido adiposo y músculo, aumento de glucólisis, gluconeogénesis e incremento de insulina $^{(26)}$, favoreciendo al incremento en la lipólisis. Sin embargo, los reportes sobre los efectos que tiene la administración de AA- $\beta$ sobre los componentes bioquímicos y hormonas sanguíneas siguen siendo inconsistentes y escasos. Las concentraciones sanguíneas de componentes hemáticos circulantes en el organismo marcan la pauta de equilibrio fisiológico y salud en el animal; sin embargo, agentes externos al organismo pueden modificar las concentraciones óptimas de estos componentes $y$, consecuentemente, llevarlos a un desequilibrio fisiológico, afectando su bienestar. Por tanto, la evaluación de estos componentes pueden ser indicadores importantes del estado 
fisiológico y patológico de los seres vivos. Los resultados de las dosis de CZ sobre las concentraciones de variables hemáticas se muestran en el Cuadro 3.

Cuadro 3: Concentraciones de componentes hematológicos en ovinos de pelo en respuesta a distintas dosis de clorhidrato de zilpaterol $\mathrm{EEM}=$ error estándar de la media; $\mathrm{L}=$ lineal; $\mathrm{C}=$ cuadrática.

\begin{tabular}{|c|c|c|c|c|c|c|c|}
\hline Variables & \multicolumn{4}{|c|}{ Dosis de CZ $\left(\mathrm{mg} \cdot \mathrm{kg}^{-1} \mathrm{de} \mathrm{PV} \mathrm{d}^{-1}\right)$} & \multirow[t]{2}{*}{ EEM } & \multicolumn{2}{|c|}{ Tendencia } \\
\hline & 0 & 0.10 & 0.20 & 0.30 & & $\mathrm{~L}$ & $\mathrm{C}$ \\
\hline $\mathrm{Gr}, \mathrm{x} 10^{12} \mathrm{~L}$ & 11.90 & 12.21 & 11.32 & 11.27 & 0.457 & 0.16 & 0.70 \\
\hline $\mathrm{Hgb}, \mathrm{g} / \mathrm{dl}$ & 10.68 & 10.67 & 10.19 & 10.25 & 0.374 & 0.26 & 0.91 \\
\hline Htc, \% & 35.60 & 36.30 & 35.00 & 34.8 & 1.15 & 0.48 & 0.68 \\
\hline $\begin{array}{l}\text { VCM, x } 10^{15} \\
\mathrm{~L}\end{array}$ & 30.21 & 30.27 & 31.30 & 31.90 & 0.870 & 0.12 & 0.78 \\
\hline HCM, Pg. & 9.10 & 8.79 & 8.98 & 9.24 & 0.205 & 0.51 & 0.17 \\
\hline $\mathrm{CCMH}, \mathrm{g} / \mathrm{dl}$ & $29.96^{\mathrm{a}}$ & $29.3^{\mathrm{ab}}$ & $28.8^{\mathrm{b}}$ & $29.2^{\mathrm{ab}}$ & 0.303 & 0.03 & $<0.05$ \\
\hline ADE, $\%$ & $\begin{array}{c}17.96 \\
\text { ab }\end{array}$ & $\begin{array}{c}18.70 \\
\mathrm{a}\end{array}$ & $18.62^{a}$ & $17.58^{b}$ & 0.375 & 0.46 & $<0.05$ \\
\hline Pqt, $\mathrm{x} 10^{9} \mathrm{~L}$ & 445.3 & 517.0 & 549.7 & 575.5 & 46.07 & $<0.05$ & 0.65 \\
\hline
\end{tabular}

Gr= glóbulos rojos; Hgb= hemoglobina; Htc= hematocrito; $\mathrm{VCM}=$ volumen corpuscular medio; $\mathrm{HCM}=$ hemoglobina corpuscular media; $\mathrm{CCMH}=$ concentración corpuscular media de hemoglobina; $\mathrm{ADE}=$ ancho de distribución de eritrocitos; Pqt= plaquetas.

${ }^{\mathrm{ab}}$ Promedios con distinta literal en la misma hilera difieren $(P<0.05)$.

La concentración corpuscular media de hemoglobina fue mayor $(P<0.05)$ en T1 que en T3, mientras que el ancho de distribución de los eritrocitos fue mayor $(P<0.05)$ en T2 y T3 que en T4. Los valores de concentración corpuscular media de hemoglobina (CMH) y ancho de distribución de eritrocitos (ADE) mostraron una tendencia cuadrática $(P<0.05)$, observándose mayor concentración de $\mathrm{CCMH}$ en los corderos no suplementados en comparación con los suplementados con Grofactor (Cuadro 3); se observó también que la concentración de ADE fue más elevada en los tratamientos 0.10 y $0.20 \mathrm{mg} / \mathrm{kg}$ en comparación con los demás tratamientos; se observó una tendencia lineal $(P<0.05)$ en los niveles de plaquetas, de tal forma que al incrementar los niveles de CZ en la dieta, las concentraciones de plaquetas incrementan. Para el resto de los componentes hematológicos no se observaron diferencias entre tratamientos $(P>0.05)$. Una posible explicación a la disminución de $\mathrm{CCMH}$ en el torrente sanguíneo es que la utilización de promotores de crecimiento reduce marcadamente la cantidad de tejido adiposo mediante la lipólisis ${ }^{(18)}$, promoviendo la vasodilatación periférica mediante la adición de ácidos grasos y glicerol al torrente sanguíneo durante este proceso, lo cual provoca el incremento en componentes hemáticos sobre el volumen plasmático ${ }^{(27)}$. Algunos estudios han mostrado que la adición de AA- $\beta$ ejerce efectos sobre algunos componentes hematológicos en rumiantes ${ }^{(19,28)}$, mediante modificaciones en sus procesos fisiológicos como vasodilatación y la acción cardiovascular ${ }^{(28)}$. Dentro de los efectos cardiovasculares que se presentan por la adición con AA- $\beta$ se encuentra el incremento de 
la frecuencia respiratoria y cardiaca de los animales. Contrariamente a lo anterior, se ha descrito que el incremento en la frecuencia respiratoria conlleva a un aumento en los niveles de los componentes de glóbulos rojos en el torrente sanguíneo ${ }^{(29)}$, debido a una alta demanda de oxígeno para activar mecanismos de disipación de calor por el incremento del calor metabólico. En este sentido, Boyd et al ${ }^{(30)}$ encontraron que la temperatura del rumen fue más baja en novillos tratados con $\mathrm{CZ}$ en relación a los testigos. Los resultados obtenidos se atribuyeron a un incremento en la frecuencia respiratoria en animales tratados, como un mecanismo para disminuir el calor acumulado por el aumento de la fermentación ruminal. Por lo anterior, los resultados de este estudio sugieren que la adición de $0.10 \mathrm{mg}$ de $\mathrm{CZ}$ incrementó la lipólisis, sin embargo, adicionar mayores dosis intensificaría la producción de calor metabólico, por lo cual el animal incrementaría la frecuencia respiratoria como mecanismo para mantener constante su temperatura corporal. No obstante, esto provocaría la vasodilatación, causando una disminución inminente de los glóbulos rojos. Resultados similares fueron reportados por Hateffi et $a l^{(19)}$, quienes encontraron que la adición de $20 \mathrm{mg} \cdot \mathrm{kg}^{-1}$ de $\mathrm{CZ}$ disminuyó los valores de hematocrito y hemoglobina, resultados que se atribuyeron al aumento de la frecuencia respiratoria. Sin embargo, los efectos de los AA- $\beta$ sobre componentes hematológicos aun no son claros debido a que existen pocos estudios con relación al tema.

$\mathrm{El}$ incremento en el promedio de plaquetas por efecto del $\mathrm{CZ}$ en la dieta pudo haberse debido a un efecto de trombocitosis sanguínea, incrementando la concentración plaquetaria como respuesta a las lesiones que puedan ocasionar el incremento de vasos sanguíneos, y al desbalance homeostático del organismo ${ }^{(16)}$. Resultados similares obtuvieron Wagner et $a l^{(24)}$ al encontrar en equinos niveles elevados de plaquetas adicionando $0.17 \mathrm{mg} \cdot \mathrm{kg}^{-1}$ de $\mathrm{CZ}$. No obstante, no se encontraron estudios en rumiantes donde se hayan evaluado los niveles plaquetarios al administrar AA- $\beta$. A pesar de lo anterior, los valores hematológicos de todas las variables obtenidas en este estudio se encontraron dentro del rango de referencia ${ }^{(31)}$. Frese et $a l^{(8)}$ realizaron un estudio para evaluar el efecto de la adición de zilpaterol y ractopamina en la dieta de bovinos en finalización sobre variables cardiovasculares, concluyendo que la adición de estos AA- $\beta$ no afectó la tasa de arritmias, aunque hubo un leve aumento en la frecuencia cardiaca, la cual, finalmente, se mantuvo dentro de los rangos de referencia señalados por la literatura para este tipo de ganado. No obstante, es importante continuar con estos estudios para comprobar si estos productos representan un riesgo, tanto para el bienestar animal como para la salud humana.

En resumen, se observaron diferencias mínimas en Col, urea, Na, así como en número de plaquetas, concentración corpuscular media de hemoglobina y ancho de distribución de eritrocitos. No obstante, todas las variables sobre hematología y bioquímica sanguínea se encontraron dentro del rango de referencia en respuesta a la adición de las dosis crecientes de clorhidrato de zilpaterol. Estos resultados sugieren que los animales experimentales no modificaron su estado acido-básico ni su homeostasis celular, además de que sus estados metabólico y fisiológico no fueron comprometidos por efecto del consumo de CZ en la dieta. Además, los resultados demuestran que no se generó un estado patológico en los 
ovinos de pelo como resultado de la adición de clorhidrato de zilpaterol (genérico) en la dieta.

\section{Agradecimientos y conflictos de interés}

Se agradece a la Secretaría de Educación Pública (Proyecto Cuerpos Académicos PRODEP, programa No. 10427) y al Fondo de Educación Superior Empresa A.C. (programa No. 10994) por el apoyo recibido para la realización del presente estudio. Los autores establecen que no existe ningún conflicto de intereses.

\section{Literatura citada:}

1. Arteaga CJD. La industria ovina en México: Memoria 1er Simposium Internacional de Ovinos de Carne. Desafíos y oportunidades para la ovinocultura en México ante los nuevos esquemas de mercado abierto. Pachuca de Soto, Hidalgo. 2003.

2. Gastélum-Delgado MA, Avendaño-Reyes L, Álvarez-Valenzuela FD, CorreaCalderón A, Meza-Herrera CA, Mellado M, Macías-Cruz U. Circannual estrous behavior in Pelibuey ewes under arid conditions of Northwestern of Mexico. Rev Mex Cienc Pecu 2015;6(1):109-118.

3. Beermann DH. ASAS Centennial paper: A century of pioneers and progress in meat science in the United States leads to new frontiers. J Anim Sci 2009;87:1192-1198.

4. Avendaño-Reyes L, Macías-Cruz U, Álvarez-Valenzuela FD, Águila-Tepato E, Torrentera-Olivera NG, Soto-Navarro SA. Effects of zilpaterol hydrochloride on growth performance, carcass characteristics, and wholesale cut yield of hair-breed ewe lambs consuming feedlot diets under moderate environmental conditions. J Anim Sci 2011;89:4188-4194.

5. Partida-de-la-Peña JA, Casaya-Rodríguez TA, Rubio-Lozano MS, Méndez-Medina RD. Effect of zilpaterol hydrochloride on the carcass characteristics of Katahdin lamb terminal crosses. Vet Méx 2015;2(2):2448-6760.

6. López-Carlos MA, Ramírez RG, Aguilera SJ, Aréchiga CF, Méndez LF, Rodríguez H, Silva JM. Effect of ractopamine hydrochloride and zilpaterol hydrochloride on growth, diet digestibility, intake and carcass characteristics of feedlot lambs. Livest Sci 2010;131(1):23-30.

7. Estrada-Angulo A, Barreras-Serrano A, Contreras G, Obregón JF, Robles-Estrada JC, Plascencia A, Zinn RA. Influence of level of zilpaterol hydrochloride supplementation on growth performance and carcass characteristics of feedlot lambs. Small Ruminant Res 2008;80(1-3):107-110. 
8. Frese DA, Reinhardt CD, Bartle SJ, Rethorst DN, Bawa B, Thomason JD, Loneragan $\mathrm{GH}$, Thomson DU. Effect of ractopamine hydrochloride and zilpaterol hydrochloride on cardiac electrophysiologic and hematologic variables in finishing steers. J Am Vet Med Assoc 2016;249:668-677.

9. FDA. New Animal Drug Application (NADA) 141-258. ZILMAX (Zilpaterol Hydrochloride) type A medicated article for cattle fed in confinement for slaughter. Approval date: 10 August, 2006.

10. Arcella D, Baert K, Binaglia M, Gervelmeyer A, Innocenti ML, Ribo O, Steinkellner $\mathrm{H}$, Verhagen $\mathrm{H}$. Review of proposed MRLs, safety evaluation of products obtained from animals treated with zilpaterol and evaluation of the effects of zilpaterol on animal health and welfare. EFSA 2016;14(9):4579.

11. Loneragan GH, Thomson DU, Scott HM. Increased mortality in groups of cattle administered the $\beta$-Adrenergic agonists ractopamine hydrochloride and zilpaterol hydrochloride. PLoS ONE 2014;9(3):e91177.

12. García, E. Modificaciones al sistema de clasificación climática de Koeppen. 3ra. ed, México, DF. Instituto de Geografía. Universidad Nacional Autónoma de México, 1985.

13. NRC. Nutrient requirements of sheep. National Academy Press, Washington, DC, 1985.

14. SAS Institute Inc.2004. SAS/STAT® User’s Guide. Cary, NC: SAS Institute Inc.

15. Radostits OM, Gay CC, Blood DC, Hinchcliff KW. Clínica Veterinária: um tratado de doenças dos bovinos, ovinos, suínos, caprinos e eqüinos. 9th ed. Guanabara Koogan, Rio de Janeiro, 2002.

16. Kaneko JJ, Harvey JW, Bruss ML. Clinical biochemistry of domestic animals. 6th ed. San Diego (USA): Academic Press; 2008.

17. Vahedi V, Towhidi A, Zare -Shahneh A, Sadeghia M, Zamanic F, Dunshea FR. Effects of $\beta$-agonist zilpaterol hydrochloride feeding and supplementation period on growth and carcass characteristics of Lori-Bakhtiari lambs. Small Ruminant Res 2014;4672:4677.

18. Bibber-Krueger CLV, Miller KA, Parsons GL, Thompson LK, Drouillard JS. Effects of zilpaterol hydrochloride on growth performance, blood metabolites, and fatty acid profiles of plasma and adipose tissue in finishing steers. J Anim Sci 2015;93:24192427.

19. Hatefi A, Towhidi A, Zali A, Zeinoaldini S, Ganjkhanlou M, Plascencia A. Effects of dietary zilpaterol hydrochloride ( $\beta 2$-agonist) supplementation on finishing castrated male goats: metabolic endocrine, blood constituents, plasma volume, respiratory rate and cardiac changes. J Appl Anim Res 2017;45(1):447-453. 
20. Zimmerli U, Blum J. Acute and longterm metabolic, endocrine, respiratory, cardiac and skeletal muscle activity changes in response to perorally administered $\beta$ adrenoceptor agonists in calves. J Anim Physiol Anim Nutr 1990;63:157-172.

21. Nourozi M, Abazari M, Raisianzadeh M, Mohammadi M, Zare-Shahne A. Effect of terbutaline and metaproterenol (two beta-adrenergic agonists) on performance and carcass composition of culled Moghani ewes. Small Ruminant Res 2008;74:72-77.

22. Chikhou FH, Moloney AP, Austin FH, Roche JF, Enright WJ. Effects of cimaterol administration on plasma concentrations of various hormones and metabolites in Friesian steers. Domest Anim Endocrinol 1991;8:471-480.

23. Buntyn JO, Steffen D, Burdick-Sanchez NC, Sieren SE, Jones SJ, Erickson GE, Carroll JA, Schmidt TB. Serum blood metabolite response and evaluation of select organ weight, histology, and cardiac morphology of beef heifers exposed to a dual corticotropin-releasing hormone and vasopressin challenge following supplementation of zilpaterol hydrochloride. J Anim Sci 2017;95:5327-5338.

24. Wagner A, Mostrom S, Hammer C, Thorson JF, Smith DJ. Adverse effects of zilpaterol administration in horses: three cases. J Equine Vet Sci 2008;28:238-243.

25. O'Connor RM, Butler WR, Finnerty KD, Hogue DE, Beermann DH. Acute and chronic hormone and metabolite changes in lambs fed the beta-agonist, cimaterol. Domest Anim Endocrinol 1991;8:537-548.

26. Dickson WM, Feldman EC, Hedge GA, Martin R, McDonald LE. Fisiología Veterinaria Cunningham. Cunningham JG editor. 3ra ed. Madrid, España: Elsevier; 2003.

27. Byrem TM, Beermann DH, Robinson TF. The beta-agonist cimaterol directly enhances chronic protein accretion in skeletal muscle. J Anim Sci 1998;76:988-998.

28. Shirato K, Tanihata J, Motohashi N, Tachiyashiki K, Tomoda A, Imaizumi K. $\beta 2$ agonist clenbuterol induced changes in the distribution of white blood cells in rats. J Pharmacol Sci 2007;104:146-152.

29. Vicente-Pérez A, Avendaño-Reyes L, Barajas-Cruz R, Macías-Cruz U, CorreaCalderón A, Vicente-Pérez R, Corrales-Navarro JL, Guerra-Liera JE. Parámetros bioquímicos y hematológicos en ovinos de pelo con y sin sombra bajo condiciones desérticas. Ecosist Recur Agropec 2018;5(14):259-269.

30. Boyd BM, Shackelford SD, Hales KE, Brown-Brandl TM, Bremer TM, Spangler ML, et al. Effects of shade and feeding zilpaterol hydrochloride to finishing steers on performance, carcass quality, heat stress, mobility, and body temperature. J Anim Sci 2015;93:5801-5811. 
31. Ahmadi-Hamedani M, Ghazvinian K, Atyabi N, Khanalizadeh P, Masoum MA, Ghodrati MS. Hematological reference values of healthy adult Sangsari sheep (Iranian fat-tailed sheep) estimated by Reference Value Advisor. J Appl Anim Res 2016;25:459-464. 